\title{
An Electronic Referral Initiative to Facilitate Referral to a Chronic Disease Self-Management Program for Persons with Transient Ischemic Attack
}

\author{
Dorothy Kessler ${ }^{1,2}$ Amir K. Afkham ${ }^{3} \quad$ Aline Bourgoin ${ }^{4}$ Sophia Gocan ${ }^{4}$ Brammiya Sivakumar ${ }^{4}$ \\ Mary E. Windsor ${ }^{5}$ Clare Liddy ${ }^{2,6}$
}

${ }^{1}$ Rotman Research Institute, Baycrest Centre for Geriatric Care, Toronto, Canada

${ }^{2}$ C.T. Lamont Primary Health Care Research Centre, Bruyère Research Institute, Ottawa, Canada

${ }^{3}$ Enabling Technologies, Champlain Local Health Integration Network, Ottawa, Canada

${ }^{4}$ Stroke Prevention Clinic, The Ottawa Hospital, Ottawa, Canada

${ }^{5}$ LHINWORKS, Winchester District Memorial Hospital,

Winchester, Canada

${ }^{6}$ Department of Family Medicine, University of Ottawa, Ottawa, Canada

Address for correspondence Dorothy Kessler, PhD, OT Reg.(Ont.), C.T Lamont Primary Health Care Research Centre, Bruyere Research Institute, Annex E, 43 Bruyere Street, Ottawa, ON, Canada K1N 5C8E (e-mail: dkessler@bruyere.org).

ACI Open 2017;1:e1-e6.

\section{Abstract}

Keywords

- electronic referral

- self-management

- transient ischemic attack

- quality improvement
Background Transient ischemic attack (TIA) is a strong predictor of subsequent stroke. Measures to decrease stroke incidence following TIA include medical management, risk factor optimization, and lifestyle modification. Best practice recommendations for stroke care promote individualized education on self-management across transitions from hospital to community settings. In the study region, patients with TIA are referred to the stroke prevention clinic where they receive rapid stroke assessment, risk factor management, education, and referral to risk reduction programs. Long-term management is typically the responsibility of primary care providers who may have limited resources for self-management support. Promotion of existing chronic disease self-management (CDSM) programs can complement this support.

Objective The objective of our project was to examine the feasibility and acceptability of an electronic referral system to an existing CDSM program to facilitate selfmanagement support for persons with TIA.

Methods We performed a descriptive evaluation of a quality improvement project that involved development and implementation of a new electronic referral (eReferral) process. A partnership between the stroke prevention clinic and the regional Living Healthy CDSM program was developed alongside a clinical information system redesign implementing an eReferral system.

Results Referral to the Living Healthy CDSM program was offered to each patient at the stroke prevention clinic. Of 912 patients seen over a 6-month period, 62 (7\%) agreed to be referred. Of these, 23 (37\%) were registered or waitlisted.

Conclusion Formation of a partnership and implementation of the eReferral system facilitated referral to the Living Healthy CDSM program. Despite low referral and enrollment rates, the eReferral system provides one option to enhance self-management support for persons with TIA. (c) 2017 Georg Thieme Verlag KG

Stuttgart · New York
License terms 


\section{Background and Significance}

Chronic disease prevention and management is an approach that has been embraced by health care organizations and systems to tackle the increasing burden of chronic diseases such as heart disease, diabetes, and stroke. In Ontario, Canada, the expanded chronic care model has been adopted as a guiding framework by the Ministry of Health and LongTerm Care to support enhanced health systems. ${ }^{1}$ Key elements within the model specific to health care organizations, such as stroke prevention clinics, include delivery system design, decision support, use of clinical information systems, an increased focused on self-care and self-management, and partnerships with community organizations and services that support patient needs. ${ }^{2}$

Transient ischemic attack (TIA) is a strong predictor of subsequent stroke, with rates ranging from 9.5 to $14.6 \%$ at 3 months. ${ }^{3,4}$ Measures to decrease the incidence of stroke following a TIA are multifaceted. Canadian Best Practice Recommendation for Stroke Care ${ }^{5}$ provides the foundation for delivery system design and decision support within Ontario stroke prevention services. This includes urgent TIA evaluation, supported by an outpatient model for rapid identification of stroke etiology and risk factor management at stroke prevention clinics. Clinical information systems such as the Stroke Report Card inform the quality and future planning of stroke service delivery.

Self-management refers to having the skills and confidence to manage daily tasks and live well with a chronic health condition. Self-management programs, such as the Stanford Chronic Disease Self-Management (CDSM) program, have been shown to contribute to slowed disease progression, reduced complications, and lowered health care costs. ${ }^{6}$ The Canadian Best Practice Recommendations for Stroke Care ${ }^{7}$ indicate that patient and family education following stroke should include self-management support. That is, they should receive individualized education including lifestyle and risk factor counseling, teaching of self-management skills, and education that is goal oriented and facilitates decision making. In addition, this education should be delivered across transitions in care so that self-management strategies are reinforced in health care and community settings. ${ }^{6}$

Despite the promising evidence for self-management programs, referral to and enrollment in these programs is low. Research studies examining factors associated with the enrollment in self-management programs have reported enrollment rates ranging from $22.8 \%{ }^{8}$ to as high as $59.6 \%{ }^{9}$ Factors associated with attendance include having better physical health, social support, older age, a non-working status, program endorsement by health care providers, encouragement from program facilitators to attend, and patient perception of low self-management support from their primary care provider. ${ }^{8-11}$ Conversely, barriers to attendance include lower income, challenges with access (transportation, scheduling, and cost), beliefs that current health care services are sufficient or the disease was not severe enough, and lack of knowledge about the purpose and structure of the program..$^{8-11}$ Measures to facilitate referral and enrollment are needed.
The use of electronic referral (eReferral) systems for clinical information delivery is growing, particularly as a way to facilitate referral to specialty and other services. ${ }^{12}$ EReferral, through electronic health records, has also been used for referrals to prevention services such as osteoporosis prevention ${ }^{13}$ and diabetes prevention programs. ${ }^{14}$ Use of eReferral systems can lead to increased referrals, efficiency in uptake of referrals, improved access to specialist care, decreased wait times, timely and high-quality communication, accurate health information transfer, and integration of health programs and services. ${ }^{12-14}$ Despite the potential benefits of eReferral systems, there are few established in Canada. ${ }^{15}$

In the Champlain region, patients with TIA are referred to the stroke prevention clinic where they receive rapid stroke assessment, medical and/or surgical interventions, stroke prevention education, individualized risk factor management, and referral to specific risk reduction programs. Long-term risk factor management and support for behavioral modification has typically been the responsibility of primary care providers who may only be able to provide limited self-management support due to competing demands. ${ }^{16}$ Promotion of existing community self-management programs for persons who have experienced TIA is potentially a cost-effective and complimentary option for extended support.

\section{Objectives}

We designed a quality improvement project to establish a partnership between a community-based CDSM program and a regional stroke prevention clinic and implemented a new clinical information system. We focused on the development and implementation of an eReferral pathway to link patients seen in the Stroke Prevention Clinic with the Living Healthy CDSM program to enhance self-management support. The objective of our project was to examine the feasibility and acceptability of the eReferral system and processes.

\section{Methods}

This is a descriptive evaluation of a quality improvement project, which involved the pilot implementation of an eReferral process, to facilitate referral to and enrollment in the Living Healthy CDSM program among patients seen at a stroke prevention clinic. We planned to examine feasibility and acceptability using program data, including referral and decision to register data, at 6 months postimplementation.

\section{Context}

\section{Stroke Prevention Clinic}

The Stroke Prevention Clinic is located at a regional stroke center. Patients attend a clinic consult visit, where they receive comprehensive stroke assessment for their presenting symptoms and a definitive diagnosis that is established by the Stroke Neurologist. Stroke risk factors are identified and the Stroke Nurse Specialist then provides individualized risk factor education and strategies for lifestyle modification. Referral to specific risk reduction programs is initiated for 
community programs such as diabetes education and smoking cessation counseling support as appropriate.

\section{Community-Based CDSM Program}

The regional Living Healthy CDSM program provides centralized enrollment to "Living a Healthy Life" workshops. The program goal is to improve access to self-management resources for people living in the region with one or more chronic conditions. The "Living a Healthy Life" workshop is a licensed program developed and researched at Stanford University ${ }^{17}$ with evidence for effectiveness in improving health outcomes and decreased health care costs. ${ }^{6}$ Trained volunteers lead in-person and on-line group workshops. Participants meet once a week for 2.5 hours, over 6 weeks. Topics covered include healthy eating, exercise, stress management, pain and fatigue management, medication management, and planning and problem solving. The program is paid for by the Ministry of Health and Long-Term Care; so, there is no cost for patients to attend. Patients or health care providers can initiate registration for a workshop.

\section{Development of the eReferral Platform}

We used an existing regional collaboration space [removed for blinded review], a secure web-based platform that enables health care workers to share information and consult with each other quickly and efficiently. The collaborative space offers a wide range of applications developed from simple team collaboration to complex business processes involving electronic forms and automated workflows. An eReferral portal was created to allow secure referral of patients from the stroke prevention clinic to the CDSM program using the regional system. The content of the form was determined by staff involved in the referral process.

The eReferral form contains patient contact information, type of chronic condition(s), and patient preference for type of workshop and means of contact (email vs. telephone). In addition, there is a space for the CDSM program administrator to enter which course a patient chose to register for, the type of class and date of enrollment after initial patient contact.

\section{Referral Process}

A new partnership was formed to promote seamless patient enrollment in the Living Healthy CDSM program. Within this pilot project, a standardized approach was developed to ensure the Stroke Nurse Specialists discuss the Living Healthy CDSM program with each patient. This includes an overview of the workshop, a discussion around options for in-class or on-line participation, provision of a brochure, and an offer to make a referral. The nurse then completes the eReferral form for those who consent to being contacted by the Living Healthy CDSM program. Once the eReferral form is submitted, automatic notification is generated to the Living Healthy CDSM staff. CDSM staffs provide one outreach call, with a message left for the patient to call back if patients from the not reached. Living Healthy CDSM staffs provide further information on program content and workshop options, and assist patients to register in upcoming workshops. Patients are put on a waitlist if an appropriate program is not currently being offered in a convenient location. This information is captured on the patients' eReferral form, and is available for viewing to both the Living Healthy CDSM and Stroke Prevention Clinic nursing staff. The referral process is displayed in - Fig. 1.

While patients have the option to self-refer and register for workshops without assistance, the eReferral process was developed to promote patient enrollment in the program through providing encouragement and support to enroll, and education about the program; thus, incorporating two factors associated with enrollment.

\section{Program Evaluation}

Data from the first 6 months of the eReferral project were examined. Number of referrals made, participant characteristics, and registration status were tracked and presented descriptively. Data were also examined for potential trends impacting registration. We were interested in the potential impact of age, gender, and home location (urban vs. rural) on decision to register and choice of an in-person or on-line program. Postal codes were used to determine whether a participant was living in a large urban center (urban) or outside

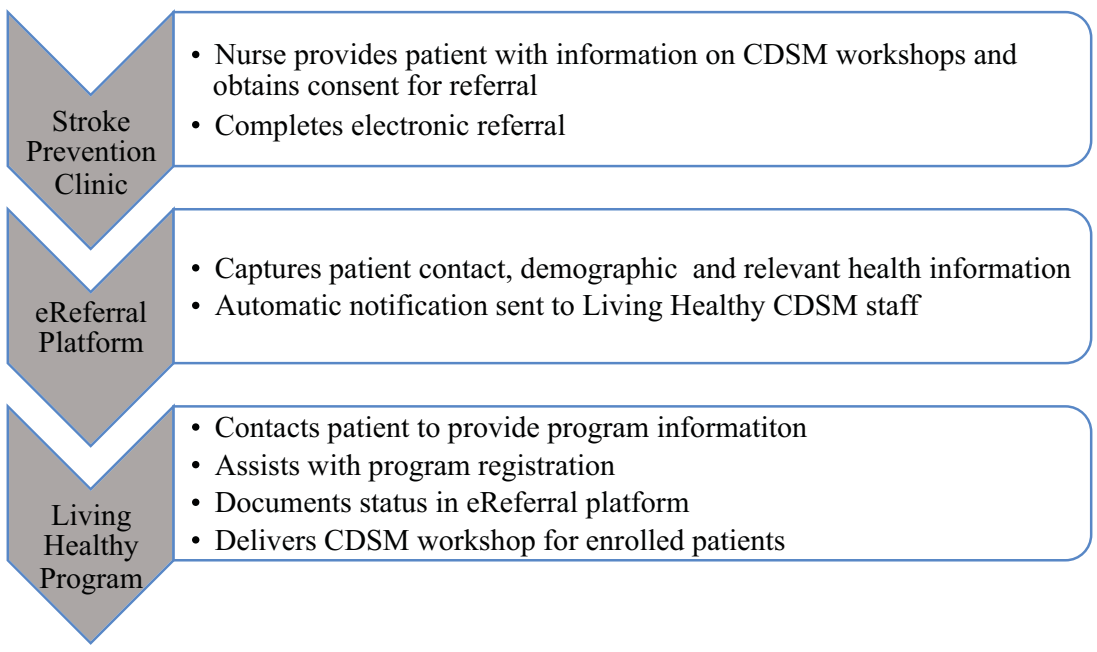

Fig. 1 Model of the eReferral process. CDSM, chronic disease self-management. 
Table 1 Referee demographics

\begin{tabular}{|l|l|}
\hline & $N(\%)$ \\
\hline Gender-male & $35(56.5)$ \\
\hline Age & $4(6.4)$ \\
\hline$<50$ & $10(16.1)$ \\
\hline $50-59$ & $19(30.6)$ \\
\hline $60-69$ & $21(33.9)$ \\
\hline$>70$ & $8(12.9)$ \\
\hline Not specified & $41(66.1)$ \\
\hline Living area & $20(32.3)$ \\
\hline Urban & $1(1.6)$ \\
\hline Rural & $42(67.7)$ \\
\hline Not specified & $20(32.3)$ \\
\hline Diagnosis & \\
\hline TIA/Stroke & \\
\hline Other &
\end{tabular}

the boundaries of this center in a smaller community (rural). To explore factors that may have impacted registration, we excluded those that could not be reached or were pending, and considered those who did not reply to the message left as having declined.

\section{Results}

Of 912 patients seen in the Stroke Prevention Clinic between April and October 2016, 62 (7\%) were referred to the Living Healthy program. Demographics data for patients referred are presented in - Table 1 .

Of the 62 patients who were referred, 23 (37\%) agreed to take part in a workshop ( 9 were registered and 14 were waitlisted), 12 (19\%) expressed interest and requested more information about the program but did not register, 23 (37\%) did not call back after a message was left, and 1 declined. Two could not be reached and one was pending contact. Among patients who agreed to take part in a workshop, 14 (61\%) had a diagnosis of TIA or stroke.

-Fig. 2 depicts registration status based on age. Patients who were older than 60 years were more likely to agree to take part in the workshop than those younger than 60 years. This tendency increased in the group older than 70 years.

\section{Program Preference}

Program preference was examined to inform future program needs for patients referred from the clinic, not the referral process itself. More patients expressed an interest in the in-person program $(15 / 23 ; 65 \%)$ than the on-line program $(8 / 23 ; 35 \%)$. However, most patients had to be waitlisted $12 / 15$ ( $80 \%$ ) for the in-person program due to lack of availability. Gender did not seem to influence preference for an in-person versus on-line program (-Table 2). While there appeared to be a preference for the in-person program among urban dwelling patients, there was no clear prefer-

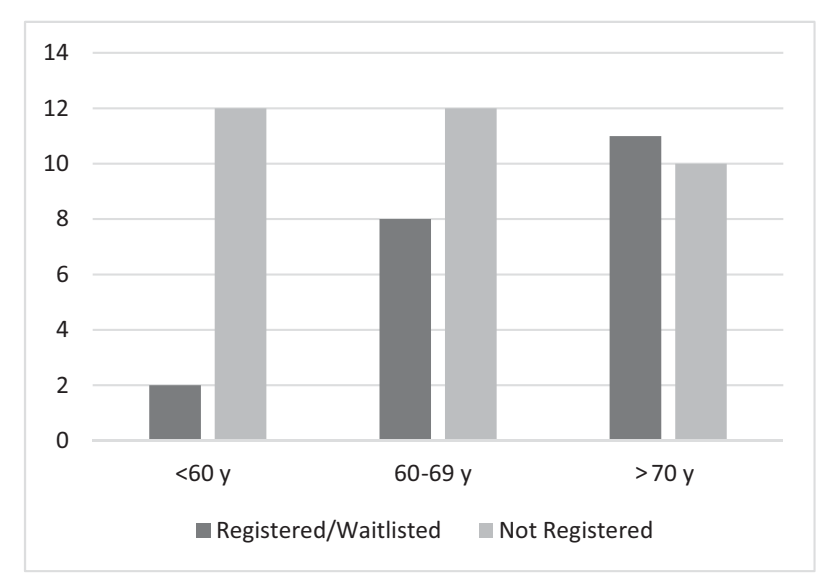

Fig. 2 Registration status by age group $(n=55)$.

ence among rural-dwelling patients. Age was not a notable factor influencing preference for on-line versus in-person programs. Preference was equal among participants aged 60 to 69 years. Among those older than 70 years, seven preferred an in-person program while four preferred the on-line format.

\section{Discussion}

We have shown that it is feasible to implement an eReferral system in a stroke prevention clinic. This health system delivery redesign facilitated a partnership with an established community resource utilizing eReferral to link interested patients with the Living Healthy CDSM program. The redesign incorporated facilitators to enrollment in self-management support programs such as endorsement by health care providers, encouragement, and support by program facilitator to enroll in the program, and information provision on the purpose of the program. ${ }^{8-11}$ As well, the program is available at no cost and is available on-line and at various locations within the region, minimizing some barriers to enrollment.

It is unclear why only $7 \%$ of those attending the Stroke Prevention Clinic agreed to be referred to the Living Healthy CDSM program. While some factors associated with enrollment were optimized, other factors may have played a role for those who declined referral. Of note, patients with dementia or living in care facilities were not approached by clinic staff but are included in the total number seen by the clinic. Tracking of number of patients approached would have provided a more accurate picture but was not implemented, as it would have

Table 2 Preference for in-person and on-line programs based on gender and home location $(n=23)$

\begin{tabular}{|l|l|l|l|}
\hline & & $\begin{array}{l}\text { In-person } \\
\boldsymbol{n}(\%)\end{array}$ & $\begin{array}{l}\text { Online } \\
\boldsymbol{n}(\%)\end{array}$ \\
\hline \multirow{3}{*}{ Gender } & Men & $8(62)$ & $5(38)$ \\
\cline { 2 - 4 } & Women & $7(70)$ & $3(30)$ \\
\hline \multirow{2}{*}{ Area } & Urban & $11(69)$ & $5(31)$ \\
\cline { 2 - 4 } & Rural & $4(57)$ & $3(43)$ \\
\hline
\end{tabular}


increased the workload of clinic staff. More detailed characteristics about patients who attended the clinic compared with those who were referred and enrolled would also help provide a more accurate picture of referral patterns.

Another consideration is that a TIA is like a stroke, producing similar symptoms, but usually lasting only a few minutes and causing no permanent damage. Without lasting symptoms, the patients' perceived severity of this event may have influenced their motivation for CDSM participation. A perception of lower disease severity has been associated with reduced participation in self-management programs. ${ }^{10}$ In addition, patients who believe that current health care is sufficient are less likely to enroll in CDSM programs. ${ }^{10}$ It is possible that patients in our study felt their health care was adequately supported through their primary care provider and/or stroke prevention clinic visit.

Of those who initially expressed an interest in the Living Healthy CDSM program, and agreed to referral, only $37 \%$ proceeded with formal registration. Notably, only one patient refused enrollment in the program when contact was made by Living Healthy staff. The enrollment rate for this project falls in the mid-range of rates found in research studies examining reasons for enrollment in self-management programs. ${ }^{8,9}$ Enrollment may be higher in research studies as opposed to actual practice, as some people may receive extramotivation to take part when they are contributing to research. The enrolment rates seen in our project are similar to those noted among persons with diabetes, where only 5 to $7 \%$ of eligible individuals participate in diabetes self-management education programs. ${ }^{18,19}$

Nineteen percent of patients referred expressed interest in the Living Healthy CDSM program but did not proceed to register. This response may reflect that these patients were at a contemplative stage of readiness for change, ${ }^{3}$ that is they were not yet prepared to take action. Readiness to make changes is an important requirement for enhancing self-management. ${ }^{20}$ Patients who are not ready to take action may need a different approach to referral that offers more support and follow-up. In this project, program staff attempted to contact patients only once. It is not clear whether further attempts to follow-up with people who did not reply to messages left would have resulted in more people registering.

Age appeared to be a factor in readiness to enroll, with those older than than 60 years being more likely to engage in the program. This may be related to having a non-working status and having more time to commit to such a program. In a study of factors associated with participation in a lifestyle program for the prevention of cardiovascular disease, unemployment status was one factor associated with higher patient enrollment in the program. ${ }^{9}$ Information on employment status was not collected for this study. Therefore, nonworking status associated with age cannot be confirmed. Research to specifically examine factors affecting readiness to enroll and actual enrolment in self-management programs is an area for further examination.

While more people were registered for the on-line program, this was influenced by the lack of available in-person sessions. All those who were waitlisted were waiting for in-person session closer to home. These findings helped inform Living Healthy program staff with regard to where programs were needed. There appeared to be a preference for in-person sessions among those living in more urban areas. The lack of a similar trend among rural dwelling patients may be related to accessibility of the on-line program in which travel was not required. Travel has been identified as a barrier to enrollment in self-management programs. ${ }^{21}$ Travel may have been less of a barrier for urban-dwelling patients. Gender did not impact preference. Adults older than 70 years are less likely to use the Internet than those who are younger. ${ }^{22}$ In this evaluation, there was a slight preference for the in-person over on-line program among participants who were older than 70 years. A larger sample size is needed to confirm this trend.

\section{Limitations}

This project was limited to examination of general factors associated with those who were referred via the eReferral process and subsequently enrolled in the Living a Healthy Life workshops. More detailed demographic data such as education and income that may have informed enrollment were not collected on the referral form, as they were not deemed necessary for the referral process by staff involved in the eReferral design. Tracking processes did not allow for identification of patients who may have self-referred after being provided with the Living Healthy CDSM program brochure. In addition, we were not able to capture more in-depth information related to patients' decisions to register or not register. This information may have provided insights into individual patient needs and how to best provide self-management support. Moreover, in this project we were not able to examine the efficacy of the Living a Healthy Life workshops for patients who decided to enroll.

\section{Conclusions}

The expanded chronic care model provides a useful framework for planning and evaluating quality improvement projects. This project focused on establishment of partnerships and clinical information system redesign. The implementation of an eReferral system was feasible and acceptable to stroke prevention clinic and Living Healthy CDSM staff. It also increased knowledge of the Living Healthy CDSM program among health care professionals and patients seen at the stroke prevention clinic. While referral and enrollment numbers were small, linking patients to the Living Healthy CDSM program using an eReferral system provided an additional avenue for self-management support for patients following TIA.

Referral and enrollment using the eReferral process was not compared with normal referral through information provision. This is an area for further investigation. Moreover, research exploring factors related to readiness of patients with TIA to engage in self-management is needed. In addition, more research is needed to evaluate the impact of selfmanagement support programs on clinical outcomes and quality of life following TIA. 


\section{Clinical Relevance}

Enrollment in a CDSM program may be facilitated through the development of partnerships between health care organizations such as the Stroke Prevention Clinic. eReferral is a feasible option to promote referral to self-management support programs.

\section{Multiple Choice Questions}

Barriers to attending a chronic disease self-management program include:

A. Not working, lower income, having better physical health.

B. Knowledge about the program, older age, social support.

C. Younger age, lower income, challenges with transportation.

D. Encouragement from health care providers, older age, lower income.

Answer $C$ is correct. People who are younger and working may not be able to commit the time to attend programs, particularly if they are during regular work hours. Challenges accessing programs are also a barrier. People who do not have easy access to transportation or are not able to afford the cost of transportation or the program will less likely to attend. People are more likely to attend a CDSM program if they receive support and encouragement to do so and they perceive the potential benefits of the program. ${ }^{8-11}$

\section{Human Subject Protections}

According to the policy activities that constitute research at the local research institutes, this project met criteria for operational improvement activities exempt from ethics review. Usual clinical practices to obtain consent and protect privacy were implemented. Data were de-identified for analysis.

Conflict of Interest

None declared.

\section{References}

1 Ministry of Health and Long-Term Care. Preventing and Managing Chronic Disease: Ontario's Framework. 2007

2 Barr VJ, Robinson S, Marin-Link B, et al. The expanded Chronic Care Model: an integration of concepts and strategies from population health promotion and the Chronic Care Model. Hosp Q 2003;7(01):73-82

3 Hill MD, Yiannakoulias N, Jeerakathil T, Tu JV, Svenson LW, Schopflocher DP. The high risk of stroke immediately after transient ischemic attack: a population-based study. Neurology 2004; 62(11):2015-2020

4 Kleindorfer D, Panagos P, Pancioli A, et al. Incidence and shortterm prognosis of transient ischemic attack in a population-based study. Stroke 2005;36(04):720-723
5 Canadian Stroke Best Practices Advisory Committee and Writing Groups. Canadian Stroke Best Practices. Heart and Stroke Foundation of Canada. Available at: http://www.strokebestpractices. ca/. Accessed June 14, 2017

6 Ory MG, Ahn S, Jiang L, et al. Successes of a national study of the Chronic Disease Self-Management Program: meeting the triple aim of health care reform. Med Care 2013;51(11):992-998

7 Cameron JI, O'Connell C, Foley N, et al; Heart and Stroke Foundation Canadian Stroke Best Practice Committees. Canadian Stroke Best Practice Recommendations: Managing transitions of care following Stroke, Guidelines Update 2016. Int J Stroke 2016;11(07):807-822

8 Dattalo M, Giovannetti ER, Scharfstein D, et al. Who participates in chronic disease self-management (CDSM) programs? Differences between participants and nonparticipants in a population of multimorbid older adults. Med Care 2012;50(12):1071-1075

9 Laws RA, Fanaian M, Jayasinghe UW, et al. Factors influencing participation in a vascular disease prevention lifestyle program among participants in a cluster randomized trial. BMC Health Serv Res 2013;13:201

10 Mahomed R, St John W, Patterson E. Factors influencing possible participation in chronic disease self-management courses. Aust J Prim Health 2006;14(03):19-26

11 Paige SR, Stellefson M, Singh B. Patient perspectives on factors associated with enrollment and retention in chronic disease selfmanagement programs: a systematic review. Patient Intell 2016; 8:21-37

12 Naseriasl M, Adham D, Janati A. E-referral solutions: successful experiences, key features and challenges-a systematic review. Mater Sociomed 2015;27(03):195-199

13 Patel VP, Mathew R, Raptis D, Christofi T, Youngman J. Overcoming barriers to secondary prevention in hip fracture patients: an electronic referral and management system for osteoporosis. Injury 2010;41(12):1249-1255

14 Chambers EC, Wylie-Rosett J, Blank AE, et al. Increasing referrals to a YMCA-based diabetes prevention program: effects of electronic referral system modification and provider education in federally qualified health centers. Prev Chronic Dis 2015;12:E189

15 Liddy C, Hogel M, Blazkho V, Keely E. The current state of electronic consultation and electronic referral systems in Canada: an environmental scan. Stud Health Technol Inform 2015;209:75-83

16 Bodenheimer T. Coordinating care-a perilous journey through the health care system. N Engl J Med 2008;358(10):1064-1071

17 Lorig K. Living a Healthy Life with Chronic Conditions: Self-management of Heart Disease, Arthritis, Stroke, Diabetes, Asthma, Bronchitis, Emphysema \& Others. Boulder, CO: Bull Publishing Company; 1994

18 Li R, Shrestha SS, Lipman R, Burrows NR, Kolb LE, Rutledge S; Centers for Disease Control and Prevention (CDC). Diabetes self-management education and training among privately insured persons with newly diagnosed diabetes-United States, 2011-2012. MMWR Morb Mortal Wkly Rep 2014;63(46):1045-1049

19 Horigan G, Davies M, Findlay-White F, Chaney D, Coates V. Reasons why patients referred to diabetes education programmes choose not to attend: a systematic review. Diabet Med 2017;34(01):14-26

20 McCusker J, Lambert SD, Cole MG, et al. Activation and selfefficacy in a randomized trial of a depression self-care intervention. Health Educ Behav 2016;43(06):716-725

21 Elzen H, Slaets JP, Snijders TA, Steverink N. Do older patients who refuse to participate in a self-management intervention in the Netherlands differ from older patients who agree to participate? Aging Clin Exp Res 2008;20(03):266-271

22 Friemel TN. The digital divide has grown old: determinants of a digital divide among seniors. New Media Soc 2016;18(02):313-331 